\title{
Properties of the hard-sphere fluid at a planar wall using virial series and molecular-dynamics simulation
}

Cite as: J. Chem. Phys. 149, 014704 (2018); https://doi.org/10.1063/1.5025332

Submitted: 08 February 2018 . Accepted: 19 June 2018. Published Online: 05 July 2018

Iván E. Paganini, Ruslan L. Davidchack (D), Brian B. Laird (D), and Ignacio Urrutia (D)
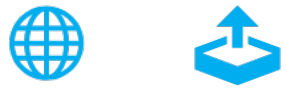

\section{ARTICLES YOU MAY BE INTERESTED IN}

Perspective: Basic understanding of condensed phases of matter via packing models The Journal of Chemical Physics 149, 020901 (2018); https://doi.org/10.1063/1.5036657

Thermodynamics of the hard-disk fluid at a planar hard wall: Generalized scaled-particle theory and Monte Carlo simulation

The Journal of Chemical Physics 149, 084701 (2018); https://doi.org/10.1063/1.5043185

Announcement: Top reviewers for The Journal of Chemical Physics 2017

The Journal of Chemical Physics 149, 010201 (2018); https://doi.org/10.1063/1.5043197 


\title{
Properties of the hard-sphere fluid at a planar wall using virial series and molecular-dynamics simulation
}

\author{
Iván E. Paganini, ${ }^{1,2}$ Ruslan L. Davidchack, ${ }^{3}$ Brian B. Laird, ${ }^{4}$ and Ignacio Urrutia ${ }^{1,2, a)}$ \\ ${ }^{1}$ Departamento de Física de la Materia Condensada, Centro Atómico Constituyentes, CNEA, \\ Buenos Aires, Argentina \\ ${ }^{2}$ Instituto de Nanociencias y Nanotecnología, INN CONICET-CNEA, Buenos Aires, Argentina \\ ${ }^{3}$ Department of Mathematics, University of Leicester, Leicester, United Kingdom \\ ${ }^{4}$ Department of Chemistry, University of Kansas, Lawrence, Kansas 66045, USA
}

(Received 8 February 2018; accepted 19 June 2018; published online 5 July 2018)

\begin{abstract}
We study the hard-sphere fluid in contact with a planar hard wall. By combining the inhomogeneous virial series with simulation results, we achieve a new benchmark of accuracy for the calculation of surface thermodynamics properties such as surface adsorption $\Gamma$ and the surface free energy (or surface tension), $\gamma$. We briefly introduce the problem of choosing a position for the dividing surface and avoid it by proposing the use of alternative functions to $\Gamma$ and $\gamma$ that are independent of the adopted frame of reference. Finally, we present analytic expressions for the dependence of system surface thermodynamic properties on packing fraction, ensuring the high accuracy of the parameterized functions for any frame of reference. The proposed parametric expressions for both, $\Gamma$ and $\gamma$, fit the accurate simulation results within the statistical error. Published by AIP Publishing. https://doi.org/10.1063/1.5025332
\end{abstract}

\section{INTRODUCTION}

The hard sphere (HS) model is one of the simplest models for interparticle interactions. Over the last century, it has played a key role in the development of physical concepts concerning the condensed matter. Numerous investigations were developed on the basis of HS models: modeling of gases, liquids, and solids as well as studies of condensation, jamming, glass transition, crystallization, defects in solids, and mixing, among others. Furthermore, HS models were instrumental in the development of modern theories of fluids. In particular, inhomogeneous fluids are frequently studied using HS as a reference system, for example, using the formalism of classical Density Functional Theory (DFT). ${ }^{1}$

Much effort has been dedicated over the decades to obtain an accurate analytic expression of the HS fluid equation of state (EOS), which describes the dependence of the bulk pressure on density. ${ }^{2-11}$ In the context of integral equations, the solution of the Percus-Yevick (PY) equation constitutes a key contribution in this direction. ${ }^{12,13}$ Different approaches to the EOS included the evaluation of virial coefficients to obtain the analytic dependence at low density and simulation, using both molecular-dynamics (MD) simulation ${ }^{9,10,14-17}$ and Monte Carlo (MC) techniques. ${ }^{18}$

In addition to the interest in determining the bulk properties, the specific properties of confined HS fluids are drawing increasing attention over time. In this case, the focus shifts to the wall-fluid surface free-energy EOS, $\gamma$, and the adsorption $\Gamma$. Seminal studies on virial series for $\gamma$ began with Bellemans and were continued by Sokołowski and Stecki. ${ }^{19-22}$ Recently,

a)iurrutia@cnea.gov.ar numerical evaluation of higher-order virial coefficients was done by Yang, Schultz, Errington, and Kofke (YSEK). ${ }^{18}$ The behavior of $\gamma$ along the complete range of densities up to freezing was studied using MD simulation by Davidchack, Laird, and Roth (DLR) ${ }^{23-27}$ Results obtained through different techniques, such as virial series, MD, MC, DFT, and scaled particle theories (SPT), have allowed for the development of analytic expressions for the dependence of $\gamma$ on density. ${ }^{25,28-31}$ However, there exist some discrepancies in recent results concerning both the behavior of $\Gamma$ and $\gamma$ at a low density, which was studied using virial series, and at higher densities, where differences exist between different studies based on MD and DFT.

In this work, we present new accurate calculations for the surface free energy and adsorption of the HS fluid at a planar hard wall. We accomplish this using a two-fold approach that combines the accurate evaluation of cluster integrals and virial coefficients combined with the results from new high resolution MD simulations. These techniques are complementary, allowing us to propose analytic expressions that accurately describe the dependence of $\gamma$ and $\Gamma$ on density. Results involve cluster integrals and virial coefficients up to seventh-order and simulation data along the complete density range of the HS fluid. In Sec. II, we describe the basic thermodynamic equations for the inhomogeneous planar systems and the power-series (virial) representation of several properties in powers of activity and packing fraction. Section III is devoted to the method used for the evaluation of cluster integrals and virial coefficients, and in Sec. IV, we describe the approach used to obtain through simulation the data of surface thermodynamics properties $\gamma$ and $\Gamma$. We present the obtained results in Sec. V, where we focus on the surface thermodynamics properties that are independent of the position of the dividing surface, which allow us to obtain accurate 
parametric expressions for surface free energy and adsorption. These expressions transform without loss of accuracy when the position of the dividing surface changes. Finally, in Sec. VI, we conclude.

\section{SURFACE THERMODYNAMICS AND ITS VIRIAL EXPANSION}

At equilibrium, the free energy of a fluid confined by a planar structureless wall at a given temperature $T$ and chemical potential $\mu$ is given by

$$
\Omega=\Omega_{\mathrm{b}}+\gamma A
$$

where $\Omega_{\mathrm{b}}=-P V$ is the bulk part of the grand potential (with $P$ as the bulk pressure and $V$ as the system volume), $A$ is the surface area, and $\gamma$ is the surface free energy. For this system, because the substrate is structureless, $\gamma$ is also equal to the fluid-wall surface tension. Both terms, surface free energy and surface tension, are used as synonyms in this work. The surface adsorption, i.e., the excess in the number of particles per unit area, is

$$
\Gamma=A^{-1} \int_{V}[\rho(\mathbf{r})-\rho] d \mathbf{r} .
$$

Here, $\rho(\mathbf{r})$ is the density distribution and $\rho$ represents the bulk fluid density. The basic relation between $\gamma$ and $\Gamma$ is the Gibbs adsorption equation, $\Gamma=-\partial \gamma / \partial \mu$. Like in Eq. (1), the average number of particles in the system (i.e., in $V$ ), $N$, also splits into bulk and surface contributions,

$$
N=N_{\mathrm{b}}+\Gamma A
$$

with $N_{\mathrm{b}}=\rho V$. The quantity $v$ is the excess volume and is defined by the relation

$$
\Gamma=-\rho v
$$

Note that $v$ is the excess volume per unit area and, therefore, has units of length. From Eq. (4), $v$ is negative if $\Gamma>0$. Like $\Gamma$, the excess volume is related to $\gamma$ through a differential relation $^{23}$

$$
v=\left(\frac{\partial \gamma}{\partial P}\right)_{T} .
$$

For this system, the excess quantities $\gamma$ and $\Gamma$ strongly depend on the chosen position for the dividing surface that defines the volume $V$ of the adopted reference region. The same applies to other excess quantities, such as $v$. However, we note that $P$ and $A$ are here independent of the adopted convention for the dividing surface. For the wall-fluid HS system, two different references are frequently adopted in the literature. One consists of setting the surface where the external potential drops from infinite to zero. The other centers around the HS diameter, $\sigma$, fixing the surface at $\sigma / 2$ inside the region where the external potential is infinite. Here the surface coincides with the position where the surface of a HS particle contacts the external wall. We call each convention I and II, respectively, and introduce the notation $\tilde{x}$ and $\bar{x}$, for quantity $x$ according to I and II. Thus, the volumes corresponding to these two references are related by

$$
(\bar{V}-\widetilde{V}) / A=\sigma / 2 .
$$

The changes in $\gamma$ and $\Gamma$ induced by the shift in the dividing surface described in Eq. (6) can be obtained by substituting Eq. (6) into Eqs. (1) and (2), respectively, giving

$$
\begin{aligned}
& \tilde{\gamma}=\bar{\gamma}-P \sigma / 2, \\
& \tilde{\Gamma}=\bar{\Gamma}+\rho \sigma / 2 .
\end{aligned}
$$

Equations (7) and (8) mix both bulk and surface thermodynamic properties in the transformation law. The corresponding transformation law for the excess volume per unit area is

$$
\tilde{v}=\bar{v}-\sigma / 2
$$

An interesting characteristic of Eq. (9) is that the transformation between $\tilde{v}$ and $\bar{v}$ represents a purely geometrical shift; i.e., it is independent of the thermodynamic state of the system. To obtain a quantity related to $\gamma$ that also transforms with a geometrical shift, we define $\alpha=\gamma / P$; then

$$
\tilde{\alpha}=\bar{\alpha}-\sigma / 2
$$

Equations (6)-(10) can be easily generalized. If we consider other two positions for the dividing surface separated by a distance $d$, the relations Eqs. (6)-(10) are still valid if we replace $\sigma / 2$ with $d$. The value of $d$ produces an offset, but is otherwise irrelevant for $v$ and $\alpha$. In this sense, we say that $v$ and $\alpha$ are independent of the adopted reference up to an arbitrary constant. The advantage of using $v$ and $\alpha$ in the place of $\Gamma$ and $\gamma$ will be highlighted in Sec. V B.

For the wall-fluid HS system, it is convenient to express $\gamma, \Gamma$, and other thermodynamic quantities in dimensionless form: $\rho^{*}=\rho \sigma^{3}, \Gamma^{*}=\Gamma \sigma^{2}, v^{*}=v / \sigma, \alpha^{*}=\alpha / \sigma, \Omega^{*}=\beta \Omega, P^{*}$ $=\beta P \sigma^{3}, \gamma^{*}=\beta \gamma \sigma^{2}$, and $\mu^{*}=\beta \mu$, where $\beta=1 / k_{\mathrm{B}} T$ is the inverse temperature (where $k_{\mathrm{B}}$ is the Boltzmann constant). In what follows, we drop the star and assume that all quantities are expressed in dimensionless form.

The thermodynamic properties of the confined fluid may be expressed as power series in the fugacity $z=\Lambda^{-3} e^{-\mu}$, where $\Lambda$ is the de Broglie thermal length, through ${ }^{31}$

$$
\Omega=-\sum_{i \geq 1} \frac{\tau_{i}}{i !} z^{i},
$$

with $\tau_{i}$ as the order $i$ cluster integral. In the bulk, we have $\frac{\tau_{i}}{i !}=b_{i} V, P=\sum_{i \geq 1} b_{i} z^{i}$, and $\rho=\sum_{i \geq 1} i b_{i} z^{i}$. Here, $b_{i}$ is the volume component of $\tau_{i}$. The coefficients $b_{i}$ are the cluster integrals in the standard (bulk) Mayer and Montroll cluster series theory. ${ }^{32-34}$ In the current case,

$$
\tau_{i}=i ! V b_{i}-i ! A a_{i},
$$

with $a_{i}$ as the planar-wall component of $\tau_{i}$. Hence, in addition to the above equations for the bulk properties, we have

$$
\gamma=\sum_{i \geq 1} a_{i} z^{i}
$$

and

$$
\Gamma=\sum_{i \geq 1} i a_{i} z^{i}
$$


TABLE I. Cluster integrals and virial coefficients of the bulk HS system up to order eight. $b_{4}$ and $B_{4}$ are known exactly. ${ }^{11}$

\begin{tabular}{lccccc}
\hline \hline$i$ & $b_{i} v_{o}^{i-1}$ & $B_{i} / v_{o}$ & $i$ & $b_{i} v_{o}^{i-1}$ & $B_{i} / v_{o}$ \\
\hline 1 & 1 & 1 & 5 & $2168.001435(4)$ & $28.224377(15)$ \\
2 & -4 & 4 & 6 & $-22362.2046(2)$ & $39.81523(10)$ \\
3 & 27 & 10 & 7 & $243676.514(4)$ & $53.342(5)$ \\
4 & $-227.4549 \ldots$ & $18.36476 \ldots$ & 8 & $-2764808.3(1)$ & $68.528(3)$ \\
\hline \hline
\end{tabular}

The coefficients $b_{i}$ are easily and accurately evaluated from the known coefficients of the pressure virial series. In terms of the packing fraction $\eta=\rho v_{0}$ (with $v_{0}=\pi / 6$ ), this series reads

$$
P=\sum_{i \geq 1} B_{i} \eta^{i}
$$

Both $b_{i}$ and $B_{i}$ are given in Table I up to eighth-order.

On the other hand, the surface tension virial series is

$$
\gamma=\sum_{i \geq 1} W_{i} \eta^{i}
$$

The coefficients $W_{i}$ are related to the cluster integrals $a_{i}$ through standard inversion and composition of power series [using Eq. (13) and $\rho$ series, below Eq. (11)]. In addition, the series representation of other surface excess properties such as $\Gamma$ and $v$ in powers of $\eta$ can also be found by usual inversion and composition of power series. Therefore, their coefficients are easily evaluated once $a_{i}$ and $W_{i}$ are known.

\section{SURFACE TERMS IN CLUSTER INTEGRALS}

Cluster integrals play a key role in the statistical mechanics of liquids. They provide an exact path to establish the relationship between the interatomic potential and the structural and thermodynamic properties of bulk and homogeneous fluids. Furthermore, cluster integrals appear naturally in systems with a fixed number of particles and confined in small cavities, even for small $N$, which are far away from the thermodynamic limit. ${ }^{35,36}$ To date, the surface area components of cluster integrals and the surface tension virial coefficients are analytically known for $n=1,2,3$. Here, we accurately evaluate the integrals for $a_{4}$ and $a_{5}$ and complement them with published data by YSEK to obtain more accurate results than in the literature for $a_{6}$ and $a_{7}$. Also, these results enable us to obtain virial coefficients $W_{n}$ up to seventhorder. To evaluate cluster integrals, we modify the method used by YSEK $^{18}$ for the activity expansion of the surface tension.

Our approach, based on simple connected cluster decomposition, is convenient because configurations compatible with simple connected clusters are simpler to generate by MC than those that are multiply connected and also because there are very few simply connected clusters for small $n$. In fact for $n=2,3,4,5,6,7$, their numbers are $1,1,2,3,6,11$, respectively. ${ }^{37}$ We give a brief general description of the adopted approach to calculate the area component of $\tau_{n}$. We consider the cluster integral of $n$ particles $\tau_{n}$ defined as the sum of all connected diagrams $\chi_{n, k}$ formed from $n$ nodes joined by $f$ bonds, where $f$ is the Mayer function $f\left(\mathbf{r}_{i j}\right)=\exp \left(-\beta \phi_{i j}\right)-1$ for particles $i$ and $j$ interacting through potential $\phi_{i j}$. Each node represents the integration of the sphere coordinate $\mathbf{r}_{i}$ over the space and is weighted by a function $g\left(\mathbf{r}_{i}\right)=g_{i}=\exp \left(-\beta \varphi_{i}\right)$, with $\varphi_{i}$ being the external potential exerted on particle $i$. The cluster integral is then

$$
\tau_{n}=\int \sum_{k} \chi_{n, k} \prod_{i=1}^{n} g_{i} d \mathbf{r}^{n} .
$$

As usual, Eq. (17) can be rearranged to give a smaller number of terms. Therefore, we join all the equivalent diagrams replacing them by a single diagram with a multiplicity factor. Also, we identify all such clusters that are simply connected, i.e., have only a single path connecting every pair of nodes as $\chi_{n, k}^{(s)}$. These are known as tree graphs. In addition, the non-simply connected clusters can be seen as a simply connected one with some extra $f$ bonds. Therefore, we factorize the integrand corresponding to each non-simply connected integral by factoring out its simply connected part as $\chi_{n, k}=\chi_{n, k}^{(s)} F_{n, k}$ (extra $f$ bonds are in $F_{n, k}$ ). We combine all the contributions with the same simply connected part to organize the integrand, which yields

$$
\tau_{n}=\int \sum_{k}^{(s)} \chi_{n, k}^{(s)} F_{n, k}^{(s)} \prod_{i=1}^{n} g_{i} d \mathbf{r}^{n},
$$

where $F_{n, k}^{(s)}$ is the sum of $F_{n, k}$ contributions corresponding to the same $\chi_{n, k}^{(s)}$. Now it is possible to select one of the nodes to play a special role and split $\prod_{1}^{n} g_{i}=g_{1}-g_{1} G_{1}$ with $G_{1}=1-\prod_{2}^{n} g_{i}$. Thus, we obtain

$$
\tau_{n}=\int g_{1} \sum_{k}^{(s)} \chi_{n, k}^{(s)} F_{n, k}^{(s)} d \mathbf{r}^{n}-\int g_{1} G_{1} \sum_{k}^{(s)} \chi_{n, k}^{(s)} F_{n, k}^{(s)} d \mathbf{r}^{n}
$$

The first term on the right-hand side of Eq. (19) is related with the bulk contribution. Here one particle is weighted by $g$ and the rest are unweighted. The second term concerns the excess inhomogeneous contribution. This is as far as we can go without introducing a dividing surface.

In the first row of Fig. 1, we present, for $n=4$, the six topologically different diagrams included in $\sum_{k} \chi_{n, k}$ at Eq. (17). Here, the multiplicity factor for each diagram is explicitly shown. Only the two graphs on the left are tree graphs; hence, Eq. (18) only involves terms: $\chi_{4,1}^{(s)}$ and $\chi_{4,2}^{(s)}$. The term $\chi_{4,1}^{(s)} F_{4,1}^{(s)}$ is drawn in the second row of Fig. 1 . There, $\chi_{4,1}^{(s)}$ is on the left of the times symbol and $F_{4,1}^{(s)}$ is on the right. We identify the common node of the three bonds of $\chi_{4,1}^{(s)}$ with the particle labeled as 1 to obtain the form in Eq. (19).

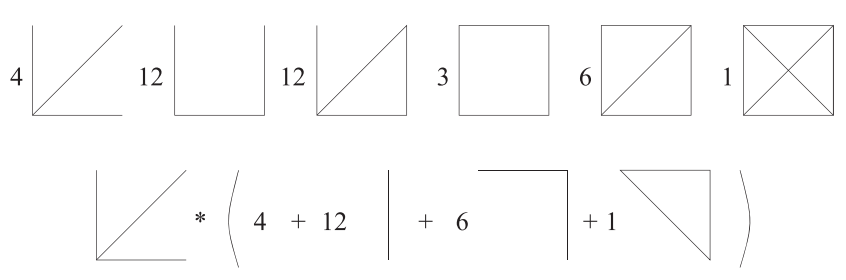

FIG. 1. A schematic representation of the cluster integrands in Eqs. (17)-(19). 
The second term in Eq. (19) can be simplified for the case of a planar hard wall. We fix the normal vector $\hat{z}$ pointing to the fluid filled region. Functions of $z$ take the following values: if $z \geq 0$, then $\varphi(z)=0$ and $g_{i}(z)=1$, but if $z<0$, then $\varphi(z)=+\infty$ and $g_{i}(z)=0$. On the other hand, $G_{1}=1$ if $z_{i}<0$ for at least one of the $i=2, \ldots, n$ particles, but $G_{1}=0$ otherwise. We introduce a dividing surface at $z=0$ to obtain

$$
n ! A a_{n}=n ! A \sum_{k}^{(s)} a_{n, k}^{(s)}=\sum_{k}^{(s)} \int g_{1} G_{1} \chi_{n, k}^{(s)} F_{n, k}^{(s)} d \mathbf{r}^{n},
$$

where we define $a_{n, k}^{(s)}$ as the part of $a_{n}$ related to $\chi_{n, k}^{(s)}$.

We employ the Mayer sampling algorithm to calculate each $a_{n, k}^{(s)}$ and use the bulk simply connected cluster integral $n ! b_{n}^{(s)}=\int \chi_{n, k}^{(s)} d \mathbf{r}^{n-1}=n !\left(2 b_{2}\right)^{n-1}$ as a reference. The first step in this approach is to fix the position of particle 1 at the origin. Next, the $n-1$ positions $\mathbf{u}_{i}$ are generated by Metropolis Monte Carlo, each of them according to the distribution $\pi=|f|$. For a given simply connected cluster $a_{n, k}^{(s)}$, we use the generated $\mathbf{u}_{i}$ to build the $n-1$ particle positions $\mathbf{r}_{i}$ according to the cluster geometry. Then, we evaluate the distances $r_{i}=\left|\mathbf{r}_{i}\right|$ and $r_{i j}=\left|\mathbf{r}_{i}-\mathbf{r}_{j}\right|$, the cluster function $\chi_{n, k}^{(s)} F_{n, k}^{(s)}$, the range of the allowed $z$ positions for particle 1 , and the length $l_{n, k}^{(s)}$. Factor $A$ in Eq. (20) comes from the integration in $d x_{1} d y_{1}$ that we have left unevaluated. The contribution related to the focused simple connected cluster is

$$
a_{n, k}^{(s)}=b_{n}^{(s)} \frac{\left\langle l_{n, k}^{(s)} \chi_{n, k}^{(s)} F_{n, k}^{(s)} / \pi^{n-1}\right\rangle_{\pi^{n-1}}}{\left\langle\chi_{n, k}^{(s)} / \pi^{n-1}\right\rangle_{\pi^{n-1}}} .
$$

Using the same set of generated positions $\mathbf{u}_{i}$, we build iteratively the particles positions $\mathbf{r}_{i}$ for each $a_{n, k}^{(s)}$ and count its contribution to the integral. We simplify the calculations by noting that the denominator is the same for each $k$, and thus we remove the index to obtain

$$
a_{n}=\frac{b_{n}^{(s)}}{\left\langle\chi_{n}^{(s)} / \pi^{n-1}\right\rangle_{\pi^{n-1}}} \sum_{k}^{(s)}\left\langle l_{n, k}^{(s)} \chi_{n, k}^{(s)} F_{n, k}^{(s)} / \pi^{n-1}\right\rangle_{\pi^{n-1}} .
$$

In particular, for HS particles, $b_{n}^{(s)}=(4 \pi / 3)^{n-1}$ and $\left\langle\chi_{n}^{(s)} / \pi^{n-1}\right\rangle_{\pi^{n-1}}=(-1)^{n-1}$.

\section{SIMULATION AND ANALYSIS}

The hard-sphere fluid next to a planar wall was simulated with molecular-dynamics using the algorithm of Rapaport. ${ }^{38}$ The hard spheres were placed between two hard walls separated by a distance $L_{z}$ along the $z$ axis, with periodic boundary conditions in the $x$ and $y$ directions. During long simulation runs, we determined the density profile of an equilibrated fluid, $\rho(z), 0 \leq z \leq L_{z} / 2$, as a function of the distance from the wall $z$, by measuring the average number of spheres in bins of width $\Delta z=0.02 \sigma$ and then averaging the result between the two walls. Between 50 and 100, statistically independent measurements were collected for each system and the statistical error determined from the variance in these measurements.
The density profile oscillates near the wall, with oscillations decaying and the density profile values converging to the bulk liquid density $\rho$ sufficiently far from the wall. To determine the excess volume $v$ from the density profile $\rho(z)$, we calculate

$$
v(z)=z-\frac{L_{z} / 2-z}{\int_{z}^{L_{z} / 2} \rho\left(z^{\prime}\right) d z^{\prime}} \int_{0}^{z} \rho\left(z^{\prime}\right) d z^{\prime}
$$

for increasing values of $z$ until $v(z)$ stops oscillating and reaches a plateau relative to the scale of the statistical errors. The value of $v(z)$ at this plateau is used as the estimator of the excess volume. At the same $z$, the expression

$$
\frac{\int_{z}^{L_{z} / 2} \rho\left(z^{\prime}\right) d z^{\prime}}{L_{z} / 2-z}
$$

is used as the estimator of the bulk liquid density $\rho$.

In order to estimate and account for possible systematic errors in our results due to the finite system size, we simulated systems with $L_{x}=L_{y} \approx 10 \sigma, 20 \sigma$, and $40 \sigma, L_{z} \approx 27 \sigma$ and $28 \sigma$ for $\eta<3.2$, and $L_{z} \approx 46 \sigma$ and $64 \sigma$ for $\eta>3.2$. We did not observe statistically the significant dependence of the results on $L_{z}$. Specifically, regression analysis with respect to $L_{z}^{-1} \mathrm{did}$ not reveal statistically significant dependence of the results on this variable. In other words, the results obtained from the systems with the same $L_{x}=L_{y}$ and different $L_{z}$ were indistinguishable within the statistical confidence intervals for all densities. In order to ensure that there was no influence of the walls on one another, we used $L_{z}$ values which were much larger than the range of observed density profile oscillations (the distance from the walls at which the oscillations become insignificant compared to the statistical error is about $9 \sigma$ at the packing fraction $\eta \approx 0.32$ and about $18 \sigma$ at the largest simulated packing fraction $\eta \approx 0.49$ ). This consideration proved sufficient to ensure that any interactions between the walls were below statistical tolerance and that size effects with respect to $L_{z}$ were insignificant compared to the statistical errors, even for high densities.

On the other hand, we did observe the dependence of the results on the system size in $x-y$ directions, but only at low densities. Specifically, linear regression applied to the results with the same $L_{z}$ and three different values of $L_{x}=L_{y}$ revealed that the linear regression model with $L_{x}^{-2}$ as the independent variable fits the data much better than the model with the independent variable $L_{x}^{-1}$. The magnitude of the slopes of the regression lines with respect to $L_{x}^{-2}$ decreased with increasing density so that the model did not demonstrate statistically the significant dependence on $L_{x}$ for $\eta \gtrsim 0.1$. In other words, the results from systems with different $L_{x}=L_{y}$ were indistinguishable within the statistical confidence intervals for $\eta \gtrsim 0.1$.

Based on these observations, the results for $v$ reported in this work were obtained by averaging the results with different $L_{z}$, applying a linear regression model as a function of $L_{x}^{-2}$ and extrapolating to $L_{x}^{-2}=0$ for $\eta<0.1$ and averaging the results with $L_{x}=L_{y} \approx 20 \sigma$ and $40 \sigma$ for $\eta>0.1$ (we did not extend the simulation of the system with $L_{x}=L_{y} \approx 10 \sigma$ to high densities). 
Once $v$ has been calculated, the surface free energy is determined by integrating Eq. (5). Because pressure is not controlled directly in the MD simulations, it is more convenient to integrate with respect to the packing fraction $\eta$ using a modified integral version of Eq. (5),

$$
\gamma(\eta)=\int_{0}^{\eta} v\left(\eta^{\prime}\right)\left(\frac{\partial P}{\partial \eta^{\prime}}\right) d \eta^{\prime} .
$$

The derivative of the pressure with respect to $\eta$ in Eq. (25) is calculated using the Kolafa, Labík, and Malijevský (KLM)low hard-sphere fluid equation of state. ${ }^{3}$ This equation of state is accurate to five decimal places over the full range of hardsphere fluid densities, and any errors generated by its use are smaller than the statistical accuracy of the current simulations. In the simulations, values of $v$ are calculated at an evenly spaced set of $\eta$ values ( $\Delta \eta=0.005240)$. Because the grid is evenly spaced, we use the extended Simpson's rule to numerically integrate Eq. (25). The values of $\gamma$ at even grid points are calculated using Simpson's rule directly on all points. For the odd grid points, the trapezoid rule is used to calculate the first integral at $\eta=0.00540$, with Simpson's rule applied for all subsequent odd grid points. To further reduce the numerical integration error, we subtract from the integrand in Eq. (25) the integrand obtained by differentiating the approximate analytic expression for $\gamma$ from the White Bear II (WBII) DFT, ${ }^{39}$

$$
\gamma=\frac{\eta\left(2+3 \eta-2 \eta^{2}\right)}{\pi(1-\eta)^{2}}-\frac{\ln (1-\eta)}{\pi} .
$$

After integration, the WBII expression for $\gamma$ from Eq. (26) is added back in to give the calculated value of $\gamma$. By subtracting this analytical approximation from the integrand, we lower the curvature of the integrand and increase the numerical accuracy of the integration scheme. In the previous work, ${ }^{25}$ we used the approximate expression for $\gamma$ from Scaled Particle theory in this process, but to ensure that the numerical integration error was smaller than the statistical error for the present high-resolution data, the more accurate WBII expression was necessary.

\section{RESULTS}

\section{A. Cluster integrals and virial coefficients}

We calculate the inhomogeneous cluster integrals $\tilde{a}_{4}$ and $\tilde{a}_{5}$ with MC. Also, $\tilde{a}_{6}$ and $\tilde{a}_{7}$ are calculated by complementing with partial results from YSEK. ${ }^{18}$ Using the exact values of $\tilde{a}_{i}$ for order $i=2,3$, the numerically obtained $\tilde{a}_{i}$ up to $i=7$ and $b_{i}$ (see Table I), we evaluate the virial coefficients $\widetilde{W}_{i}$ and $\bar{W}_{i}$ for $i=4,5,6,7$. In Table II are shown our results for the cluster integrals and virial coefficients of the surface tension series using convention I. We also include data from YSEK ${ }^{18}$ for comparison. There one can observe small but significant differences between results of the present work (PW) and those from the previous literature. Notice that some of these small differences correspond to the comparison with the exact results, for $n=2$, 3 . It is interesting to mention that most of the significant differences of $\widetilde{W}_{n}$ between our results and those of YSEK disappear when we consider the exact values of $\widetilde{W}_{2}$ and $\widetilde{W}_{3}$ and use MC partial results in Ref. 18 to recalculate higher-order $\widetilde{W}_{n}$. In fact,
TABLE II. Coefficients of the power series for $\gamma$ up to $i=7$ employing convention I for the dividing surface position. The two columns on the left correspond to the series in powers of $z$, while those on the right correspond to powers of packing fraction $\eta$ (virial series). Besides, $\tilde{a}_{1}=\widetilde{W}_{1}=0$.

\begin{tabular}{lcrcc}
\hline \hline$n$ & $\tilde{a}_{n} \mathrm{PW}$ & $\tilde{a}_{n} \mathrm{YSEK}$ & $\widetilde{W}_{n} \mathrm{PW}$ & $\widetilde{W}_{n} \mathrm{YSEK}^{18}$ \\
\hline $2^{\mathrm{a}}$ & $-0.39270 \ldots$ & $0.39269(1)$ & $-1.43239 \ldots$ & $-1.43229(7)$ \\
$3^{\mathrm{b}}$ & $2.41453 \ldots$ & $-2.4146(1)$ & $-6.09791 \ldots$ & $-6.1004(14)$ \\
4 & $-14.360788(6)$ & $14.354(2)$ & $-13.69479(8)$ & $-13.720(12)$ \\
5 & $87.60987(3)$ & $-87.60(3)$ & $-22.290(2)$ & $-22.412(8)$ \\
6 & $-548.818(4)$ & $548.9(3)$ & $-32.01(19)$ & $-32.51(4)$ \\
7 & $3529.0(2)$ & $-3530(3)$ & $-63(17)$ & $-65(17)$ \\
\hline \hline
\end{tabular}

$\mathrm{a} \tilde{a}_{2}=-\pi / 8, \widetilde{W}_{2}=-9 / 2 \pi$.

$\mathrm{b} \tilde{a}_{3}=137 \pi / 560, \widetilde{W}_{3}=-1341 / 70 \pi$.

this is the case for the first non-exactly known virial coefficient producing $\widetilde{W}_{4}=13.70(1)$. This integral was evaluated for the first time forty years ago by Stecki and Sokołowski; ${ }^{21}$ they obtained $\widetilde{W}_{4}=14.045(72)$.

The virial series coefficients of the surface tension using convention II are shown in Table III. We observe that improved precision of the present work results for $\bar{W}_{4}$ and $\bar{W}_{5}$ and relatively significant differences from the previously published values. These small differences could be related to the small inaccuracies of the YSEK results discussed in Table II. The precision diminishes at sixth and seventh-order. A comparison between the approximate virial series coefficients using both conventions I and II shows that absolute uncertainties are the same because the error introduced by virial coefficients $B_{i}$ is much smaller.

\section{B. MD and the analytic expressions for the surface thermodynamics properties}

Here, we present the simulation results for the surface properties. Numerical data obtained using MD can be found in the supplementary material. In addition, based on the obtained virial series coefficients and the simulation results, we look for analytic parameterized expressions that accurately describe $\gamma(\eta)$ and $\Gamma(\eta)$. Before proceeding, we should fix the convention for $V$ (and consequently for the surface properties). Naturally, once we adopt a convention and find analytic expressions that fit the surface properties of the system, the thermodynamic description becomes less universal because, given the

TABLE III. Coefficients of the surface tension series in powers of the packing fraction. Here, the convention II for the position of the dividing surface is used. PW results are in the second column while the third column corresponds to YSEK and DLR. ${ }^{18,25}$

\begin{tabular}{lll}
\hline \hline$n$ & $\bar{W}_{n}$ PW & $\bar{W}_{n}$ YSEK-DLR \\
\hline $1^{\mathrm{a}}$ & $0.95493 \ldots$ & $0.95493 \ldots$ \\
2 & $2.38732 \ldots$ & $2.38732 \ldots$ \\
3 & $3.45139 \ldots$ & $3.45139 \ldots$ \\
4 & $3.84227(8)$ & $3.817(12)$ \\
5 & $4.6622(3)$ & $4.54(8)$ \\
6 & $6.01(19)$ & $5.5(4)$ \\
7 & $-12(17)$ & $-14(17)$ \\
\hline \hline
\end{tabular}

${ }^{\mathrm{a}} \bar{W}_{1}=3 / \pi, \bar{W}_{2}=15 / 2 \pi$, and $\bar{W}_{3}=759 / 70 \pi$. 
approximate nature of the description, certain loss of accuracy occurs when we transform to a different convention. This is particularly problematic for the transformation of $\gamma(\eta)$ which adds a term proportional to $P$, a quantity that we only know approximately. This means that a good fitting function under a certain convention may transform into a worse one under another.

In Ref. 31, this issue was dealt with the study of fitting functions adopting both conventions I and II. Here, to overcome the problem, we change the focus from $\gamma(\eta)$ and $\Gamma(\eta)$ to quantities $\alpha(\eta)$ and $v(\eta)$, which transform trivially by changing the ordinate at the origin but fixing its shape. In other words, the shape of both $\alpha(\eta)$ and $v(\eta)$ is invariant under a translation of the dividing surface, which is not true for $\gamma(\eta)$ and $\Gamma(\eta)$. To obtain $\alpha$ (and also to transform between different conventions), we use the KLM-low EOS. $^{3}$

For the excess volume, we propose the following expression based on a modified virial series:

$$
\begin{aligned}
v= & \frac{1}{2}-\frac{3}{2} \eta+\frac{339}{140} \eta^{2}+a_{1} \eta^{3}+a_{2} \eta^{4}+a_{3} \eta^{5} \\
& +a_{4} \eta^{6}\left(\frac{1}{2}-\eta\right)^{-1}+a_{5} H .
\end{aligned}
$$

Here, $H=h-S_{3}(h)$ and $h=\left(\frac{1}{2}-\eta\right) \ln (1-2 \eta)$, and also $S_{i}(h)$ is the order $i$ McLaurin series of $h$ [i.e., $S_{3}(h)=-\eta+\eta^{2}+\frac{2}{3} \eta^{3}$ ]. Equation (27) has the right terms up to order $\eta^{2}$, and the fitting coefficients have values $a_{1}=-843 / 280, a_{2}=111 / 35$, $a_{3}=-121 / 84, a_{4}=-3 / 1120$, and $a_{5}=-283 / 1400$. The coefficients of the power series in $\eta$ are shown in Table IV where the zero abscissa value is omitted because it is irrelevant. The excess volume $v$ is shown in Fig. 2 where we plot the MD data and the analytic expression Eq. (27). Note that on the scale of the graph, the curve fits the data very well. To verify the accuracy of Eq. (27), we plot in Fig. 3 the difference between $v$ from diverse sources and the present work MD results. For comparison, a selection of older MD data and existing analytic expressions are presented (taken from Refs. 25 and 31. See also Ref. 40). We observe that MD results in PW (with statistical errors smaller than $10^{-4}$ ) are more accurate than those published and are not followed by existing formulae for $v$. Meanwhile, Eq. (27) reproduces very well the MD results within the statistical error. By construction, fitting MD data for $v$ guarantee the accuracy of Eq. (27) times $\rho$ in fitting $\Gamma$. This point is analyzed in detail for the case of $\alpha$ and $\gamma$.

TABLE IV. Virial coefficients of $v$ and $\alpha$ (i.e., those of their series representation in powers of $\eta$ ).

\begin{tabular}{lllll}
\hline \hline Ord & \multicolumn{1}{c}{$v$} & Equation (27) & $\alpha$ & Equation (28) \\
\hline 1 & -1.5 & -1.5 & -0.75 & -0.75 \\
2 & $-2.4214 \ldots$ & $-2.4214 \ldots$ & $-0.1928 \ldots$ & $-0.1928 \ldots$ \\
3 & $-3.0537(2)$ & -3.0107 & $1.10085(4)$ & 1.1137 \\
4 & $3.620(7)$ & 3.0366 & $-0.372(1)$ & -0.52 \\
5 & $-4.1(6)$ & -1.6022 & $1.6(1)$ & -1.0 \\
\hline \hline
\end{tabular}

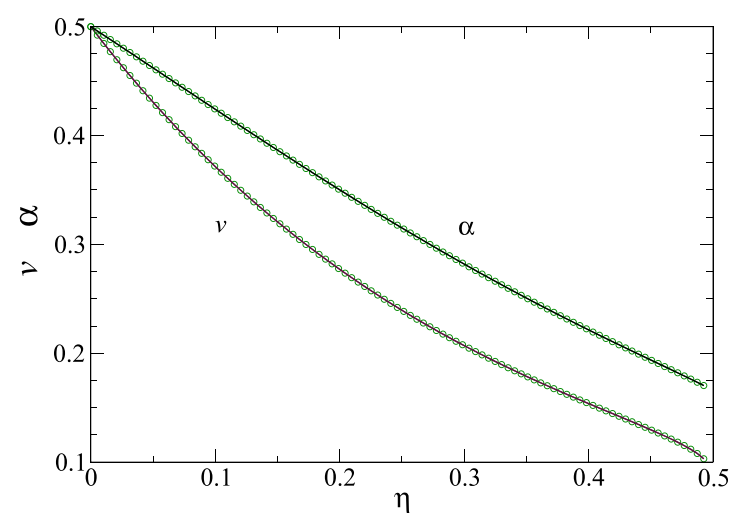

FIG. 2. Excess volume (bottom) and $\alpha$ ratio (top) results. Circles denote the MD data while continuous lines show the parametric representations for $v$ and $\alpha$, Eqs. (27) and (28), respectively. The errors in the MD data are considerably smaller than the symbols, as can be appreciated in Figs. 3 and 4.

For $\alpha$, we find that the expression based on a modified virial expansion

$$
\alpha=\frac{1}{2}-\frac{3}{4} \eta-\frac{27}{140} \eta^{2}+a_{1} \eta^{3}+a_{2} \eta^{4}+a_{3} \eta^{5}\left(\frac{1}{2}-\eta\right)+H,
$$

describes accurately the MD data. Here $H=h-S_{2}(h)$, $h=\left[a_{4}\left(\frac{1}{2}-\eta\right)+a_{5}\left(\frac{1}{2}-\eta\right)^{2}\right] \ln (1-2 \eta)$ and $S_{2}(h)$ $=-\left(a_{4}+a_{5} / 2\right) \eta+\left(a_{4}+3 a_{5} / 2\right) \eta^{2}$. Again, we reproduce terms up to order $\eta^{2}$, as can be seen in Table IV, while the order $\eta^{3}$ coefficient has an error of $1.5 \%$. The fitting parameters are $a_{1}=227 / 140, a_{2}=-369 / 1400, a_{3}=-177 / 112, a_{4}=-11 / 700$, and $a_{5}=731 / 980$. In Fig. 2, the dependence of $\alpha$ on $\eta$ is plotted. There, the agreement between MD results and analytic expression (28) is visible in the scale of the plot. To verify the quality of our results, we plot in Fig. 4 the difference between Eq. (28) and the MD data, where zero corresponds to the reference (MD results) plotted with its statistical error. We can note two features. First, the high accuracy of MD data with error bars $\lesssim 10^{-5}$. Second, the points derived from the fitting expression Eq. (28) describes adequately the MD results (within the statistical uncertainty). In the inset, plotted

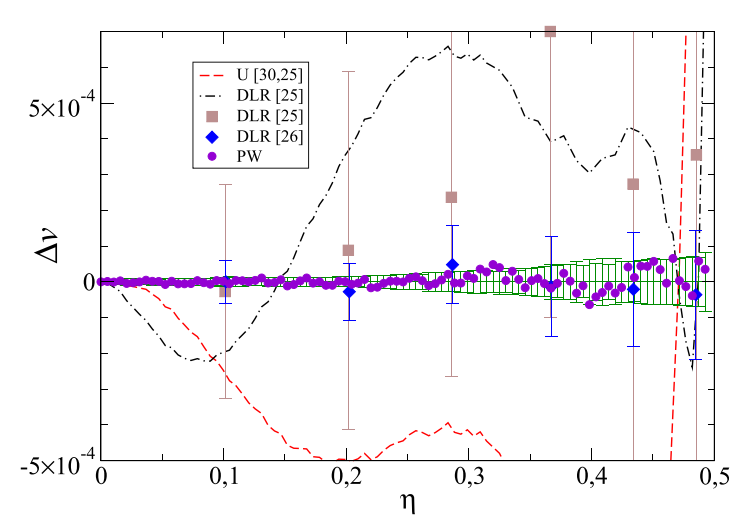

FIG. 3. Deviations of the previous simulation results and parameterizations of $v$ from the PW high-precision MD results. Error bars in green show the statistical error of MD results in PW, and squares and diamonds show published MD data. Circles correspond to Eq. (27), and lines correspond to previous analytic expressions (see the text). 


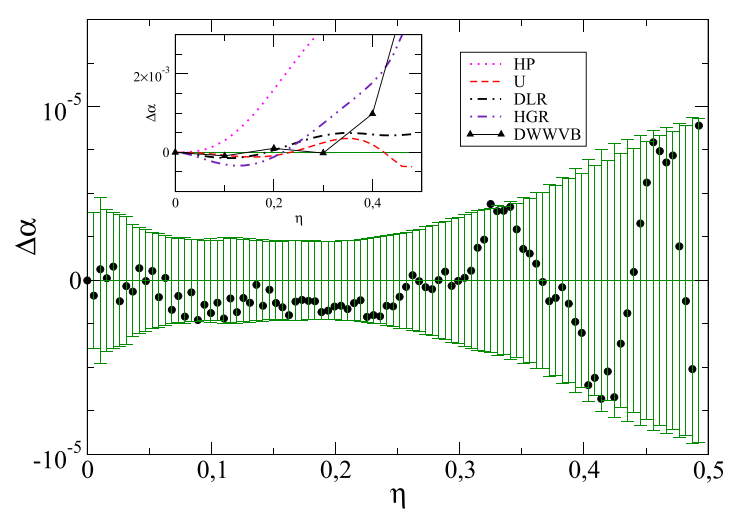

FIG. 4. Deviation from simulation data for $\alpha$. Error bars show the statistical error of MD results, and circles correspond to Eq. (28). Other lines in the inset are explained in the text.

in a smaller scale, we compare with different analytic expressions. Those are the fitting expressions from Henderson and Plischke $^{29}$ (HP, dotted), Urrutia ${ }^{31}$ (U, dashed), DLR ${ }^{25}$ (dotteddashed), and the DFT-WBII based expression by HansenGoos and Roth ${ }^{39}$ (HGR, dotted-dotted-dashed). Also, results obtained by direct minimization of DFT-WBII by Deb et al. ${ }^{27}$ (DWWVB; from Table I, col. 3, therein) are drawn with triangles. We note that the collected data for $\alpha$ from different sources show a deviation much larger than the statistical error of the highly accurate MD results, in particular, for $\eta>0.3$. By construction, the analytic expressions for the surface tension (adsorption) must be as accurate as the expression for $\alpha(v)$, i.e., if the expression for $\Delta \alpha$ produces results that are within the statistical error, then the same applies to surface tension.

In Figs. 5 and 6 are shown the results for $\gamma=\alpha P$ in frames of reference I and II, respectively. The errors of the plotted MD data are smaller than $10^{-4}$. This would make them invisible in the scale of the plots and thus error bars are not drawn. In both cases, we observe that formulae based on Eqs. (28) and (10) fit very well the simulation results.

To appreciate the precision of the new high-resolution MD results and the goodness of the obtained expression for $\gamma$, we draw in Fig. 7 the difference of several sources for $\gamma$, including our analytic expression, from the presented MD results. Note that $\Delta \gamma=\Delta \tilde{\gamma}=\Delta \bar{\gamma}$. For comparison, previously published

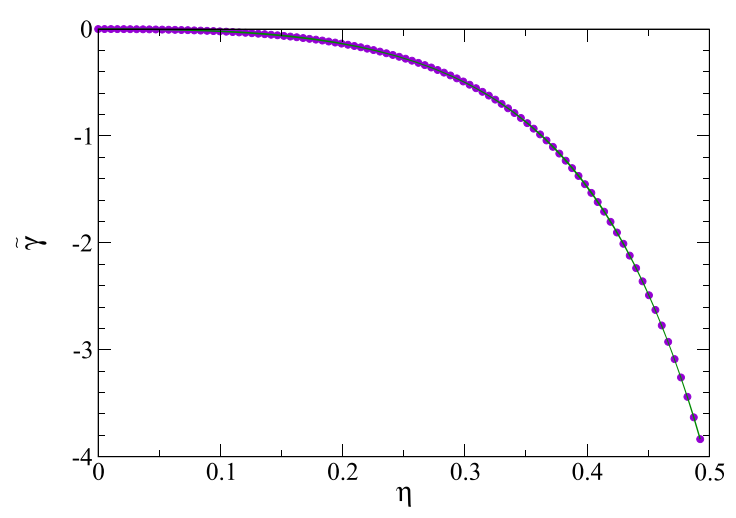

FIG. 5. MD results and fitting function for $\tilde{\gamma}$, in circles and continuous line, respectively.

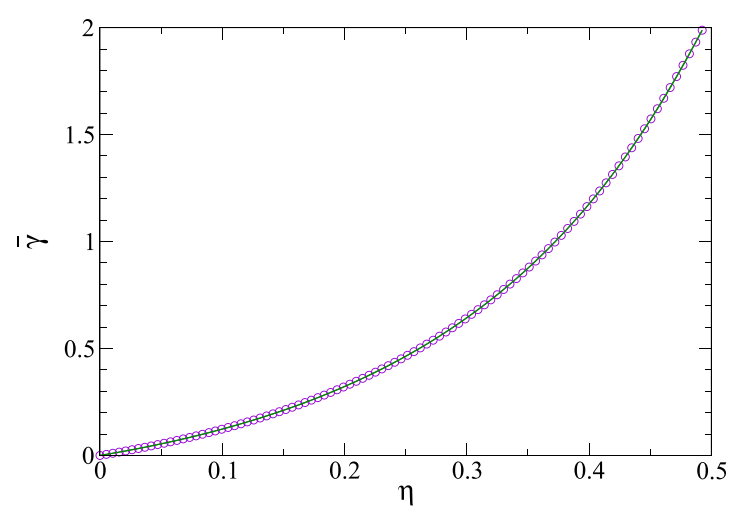

FIG. 6. MD results and fitting function for $\bar{\gamma}$.

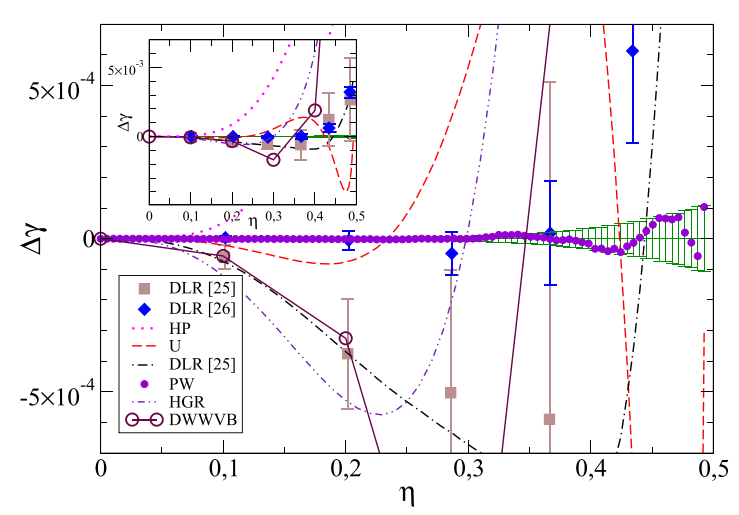

FIG. 7. Deviation from simulation data for $\gamma$. Older MD results are taken from Refs. 25 and 26 (squares and diamonds, respectively). Closed circles are derived from Eq. (28). Open circles and lines are explained in the text.

MD data from DLR ${ }^{25,26}$ are also plotted. ${ }^{41}$ Closed circles are based on Eq. (28). Data from the different analytic expression for $\gamma$ are presented with lines. They correspond to HP, ${ }^{29}$ dotted, $\mathrm{U},{ }^{31}$ dashed, DLR, ${ }^{25}$ dotted-dashed, and the DFT-WBII expression obtained by HGR, ${ }^{39}$ dotted-dotted-dashed, respectively. Also, a different set of DFT-based results obtained by DWWVB $^{27}$ (see Table I, col. 3, therein) is drawn with open circles. We note a large dispersion between different sources, particularly for $\eta \gtrsim 0.4$. In the inset, the large dispersion of the different sources for $\gamma$ is apparent also in the smaller scale.

We note that the presented MD results are much more precise than previous studies and that there are significant differences between PW and those of Refs. 25 and 26. This difference is mainly attributed to the high precision and the small separation $\Delta \eta$ of the MD results for $v$ and also to the use of the WBII expression to reduce the integration error. We verify that Eq. (28) reproduces quite well the MD results. Other analytic expressions do not follow the new high accuracy MD results for $\gamma$.

\section{CONCLUDING REMARKS}

In this work, the system of a hard-sphere (HS) fluid at a planar hard wall was studied and new high precision results were presented. The results include cluster integrals [solved by Monte Carlo (MC)], virial series coefficients, and 
Molecular-Dynamics (MD) simulation results for the surface thermodynamic properties of the system. On one hand, cluster integrals and virial series coefficients allow an analytic description of the low-density behavior of the system. On the other hand, simulation results are important in order to obtain accurate numerical results for the surface thermodynamics along the entire range of densities of the HS fluid. The use of both virial series coefficients and simulation results makes it possible to obtain very accurate parametric expressions describing the properties of the fluid-wall interface. We adopt a point of view independent of the reference frame by parameterizing $v(\eta)$ (the excess surface volume) and $\alpha(\eta)=\gamma / P$ in the place of adsorption and surface tension (or surface free energy). This independence implies that a change of reference only produces a trivial shift of the ordinate at the origin, which ensures the high accuracy of fitting functions for any frame of reference that could be adopted. This goal extends to the derived expressions for adsorption and surface tension.

With respect to the cluster integrals and virial coefficients, a question about the possibility of obtaining an analytic value for $\widetilde{W}_{4}$ (analogously $\bar{W}_{4}$ and $\tilde{a}_{4}$ ) still remains open given that the bulk virial coefficient $B_{4}$ is exactly known. The exact calculation of $\widetilde{W}_{4}$ is a difficult task because it involves a nine-fold integral. For comparison, we can mention that $B_{4}$ involves a six-fold integral, while $B_{5}$, which is not analytically known, is also a nine-fold integral. Another open question is the possibility of direct calculation using MC of the sixth and seventhorder coefficients, which surely would improve their accuracy. To extend our approach to higher-orders, the decomposition of $\tilde{a}_{6}$ and $\tilde{a}_{7}$ in terms of simply connected clusters must be performed.

This study of the HS fluid in contact with a planar hard wall, involving a very accurate description of fluid/wall thermodynamics properties, is a necessary first step for future developments concerning the HS fluid in contact with curved walls, in particular, for the search of an accurate description of the curvature terms in its free energy.

\section{SUPPLEMENTARY MATERIAL}

See supplementary material for the table with the MD results.

\section{ACKNOWLEDGMENTS}

Ignacio Urrutia and Iván E. Paganini acknowledge support from Argentina Grant No. CONICET PIP-112-201501-00417. Brian B. Laird acknowledges support from the US National Science Foundation (Grant No. CHE-1465226). Ruslan L. Davidchack did part of the work during a study leave granted by the University of Leicester. The molecular dynamics simulations were performed on the ALICE
High Performance Computing Facilities at the University of Leicester.

${ }^{1}$ Theory of Simple Liquids, 4th ed., edited by J.-P. Hansen and I. R. McDonald (Academic Press, Oxford, 2013), p. 636.

${ }^{2}$ N. F. Carnahan and K. E. Starling, J. Chem. Phys. 51, 635 (1969).

${ }^{3}$ J. Kolafa, S. Labík, and A. Malijevský, Phys. Chem. Chem. Phys. 6, 2335 (2004).

${ }^{4}$ A. Mulero, C. Galán, M. Parra, and F. Cuadros, in Theory and Simulation of Hard-Sphere Fluids and Related Systems, Lectures Notes in Physics, edited by A. Mulero (Springer, Berlin, 2008), Vol. 753, Chap. 3, pp. 37-60.

${ }^{5}$ M. N. Bannerman, L. Lue, and L. V. Woodcock, J. Chem. Phys. 132, 084507 (2010).

${ }^{6}$ A. Santos, "Exact solution of the Percus-Yevick approximation for hard spheres ... and beyond," in A Concise Course on the Theory of Classical Liquids: Basics and Selected Topics (Springer International Publishing, Cham, 2016), Chap. 7, pp. 203-253.

${ }^{7}$ H. Hansen-Goos, J. Chem. Phys. 144, 164506 (2016).

${ }^{8}$ N. Metropolis, A. W. Rosenbluth, M. N. Rosenbluth, A. H. Teller, and E. Teller, J. Chem. Phys. 21, 1087 (1953).

${ }^{9}$ E. J. J. van Rensburg, J. Phys. A: Math. Gen. 26, 4805 (1993).

${ }^{10}$ S. Labík, J. Kolafa, and A. Malijevský, Phys. Rev. E 71, 021105 (2005).

${ }^{11}$ A. J. Schultz and D. A. Kofke, Phys. Rev. E 90, 023301 (2014).

${ }^{12}$ E. Thiele, J. Chem. Phys. 39, 474 (1963).

${ }^{13}$ M. S. Wertheim, Phys. Rev. Lett. 10, 321 (1963).

${ }^{14}$ M. N. Bannerman, R. Sargant, and L. Lue, J. Comput. Chem. 32, 3329 (2011).

${ }^{15}$ J. Delhommelle and D. J. Evans, J. Chem. Phys. 114, 6229 (2001).

${ }^{16}$ K. W. Kratky, J. Stat. Phys. 29, 129 (1982).

${ }^{17}$ F. H. Ree and W. G. Hoover, J. Chem. Phys. 46, 4181 (1967).

${ }^{18}$ J. H. Yang, A. J. Schultz, J. R. Errington, and D. A. Kofke, J. Chem. Phys. 138, 134706 (2013).

${ }^{19}$ A. Bellemans, Physica 28, 493 (1962).

${ }^{20}$ S. Sokołowski, Czech. J. Phys. 27, 850 (1977).

${ }^{21}$ J. Stecki and S. Sokołowski, Phys. Rev. A 18, 2361 (1978).

${ }^{22}$ S. Sokołowski and J. Stecki, Acta Phys. Pol. A 55, 611 (1979).

${ }^{23}$ R. L. Davidchack, J. Chem. Phys. 133, 234701 (2010).

${ }^{24}$ B. B. Laird and R. L. Davidchack, J. Chem. Phys. 132, 204101 (2010).

${ }^{25}$ R. L. Davidchack, B. B. Laird, and R. Roth, Mol. Phys. 113, 1091 (2015).

${ }^{26}$ R. L. Davidchack, B. B. Laird, and R. Roth, Condens. Matter Phys. 19, 23001 (2016); e-print arXiv:1603.06906.

${ }^{27}$ D. Deb, A. Winkler, M. H. Yamani, M. Oettel, P. Virnau, and K. Binder, J. Chem. Phys. 134, 214706 (2011).

${ }^{28}$ H. Reiss, H. L. Frisch, E. Helfand, and J. L. Lebowitz, J. Chem. Phys. 32, 119 (1960).

${ }^{29}$ D. Henderson and M. Plischke, Proc. R. Soc. London A 410, 409 (1987).

${ }^{30} \mathrm{H}$. Hansen-Goos and R. Roth, J. Chem. Phys. 124, 154506 (2006).

${ }^{31}$ I. Urrutia, Phys. Rev. E 89, 032122 (2014).

${ }^{32}$ J. E. Mayer and E. Montroll, J. Chem. Phys. 9, 2 (1941).

${ }^{33}$ J. E. Mayer and M. G. Mayer, Statistical Mechanics (Wiley, New York, 1940).

${ }^{34}$ T. L. Hill, Statistical Mechanics (Dover, New York, 1956).

${ }^{35}$ I. Urrutia, J. Chem. Phys. 135, 024511 (2011).

${ }^{36}$ I. Urrutia, J. Chem. Phys. 135, 099903 (2011).

${ }^{37}$ See http://oeis.org/A000055 for integer series.

${ }^{38}$ D. C. Rapaport, The Art of Molecular Dynamics Simulation, 2nd ed. (Cambridge University Press, 2004).

${ }^{39}$ H. Hansen-Goos and R. Roth, J. Phys.: Condens. Matter 18, 8413 (2006).

${ }^{40}$ In particular, see Eqs. (20) and (28) in Ref. 25. The expression for $\Gamma$ taken from Eq. (28) in Ref. 25 was multiplied by $\pi^{-1}$ to correct a misprint.

${ }^{41}$ Three points corresponding to high packing fraction lying at $(0.4338$, $-0.0013),(0.4854,-0.0028)$ (squares) and $(0.4850,-0.0033)$ (diamonds) are outside of the plot range in Fig. 7. 